\title{
Usual dietary treatment of gestational diabetes mellitus assessed after control diet in randomized controlled trials: subanalysis of a systematic review and meta-analysis
}

\author{
Apolonia García-Patterson ${ }^{1}$ - Montserrat Balsells ${ }^{2}$ Jennifer M. Yamamoto ${ }^{3}$ Joanne E. Kellett ${ }^{4}$. Ivan Solà ${ }^{1,5,6}$. \\ Ignasi Gich ${ }^{6,7,8,9}$. Eline M. van der Beek ${ }^{10,11}$. Eran Hadar ${ }^{12}$. Eurídice Castañeda-Gutiérrez ${ }^{13} \cdot$ Seppo Heinonen $^{14,15}$. \\ Moshe Hod ${ }^{12}$. Kirsi Laitinen ${ }^{16,17}$. Sjurdur F. Olsen ${ }^{18}$ • Lucilla Poston ${ }^{19}$. Ricardo Rueda ${ }^{20}$ • Petra Rust ${ }^{21}$. \\ Lilou van Lieshout ${ }^{22} \cdot$ Bettina Schelkle ${ }^{22} \cdot$ Helen R. Murphy ${ }^{4,23,24} \cdot$ Rosa Corcoy $^{25,26,27}$
}

Received: 8 August 2018 / Accepted: 26 September 2018 / Published online: 17 October 2018

(c) The Author(s) 2018

\section{Abbreviations \\ DRI Dietary reference intakes \\ GDM Gestational diabetes mellitus \\ RCT Randomized controlled trial}

\section{Introduction}

The prevalence of GDM is on the rise in relation to an increase in predisposing maternal characteristics. The increase is more marked with application of IADPSG-WHO 2013 criteria [1], with very high rates in special populations [2].

Lifestyle modifications are the first step in the management of GDM and medical nutrition therapy is an essential component of it. Maternal diet should provide adequate energy intake to promote maternal and fetal health, help achieve glycemic goals and be culturally appropriate and individualized [3]. DRI for normal weight pregnant women should be taken into account: provide no increase in energy requirement during the first trimester, $+340 \mathrm{kcal} /$ day in the second trimester and $+452 \mathrm{kcal} /$ day in the third; provide $>=175 \mathrm{~g}$ carbohydrate/day, $71 \mathrm{~g}$ protein/day and $28 \mathrm{~g}$ fiber/day; and have an acceptable energy macronutrient distribution range (45-65\% of energy from carbohydrates, $20-35 \%$ of energy from fat, $10-35 \%$ of energy from protein).

Managed by Antonio Secchi.

Helen M. Murphy and Rosa Corcoy, senior authors, contributed equally.

Rosa Corcoy

rcorcoy@santpau.cat

Extended author information available on the last page of the article
However, little is known about the characteristics of diets consumed by women with GDM.

We aimed to characterize the dietary intake of women with GDM in usual clinical care.

\section{Study protocol}

We recently performed a systematic review and meta-analysis on RCTs addressing modified dietary interventions for the treatment of GDM and providing information on maternal glycemic control and birthweight-related variables [4] (published protocol: PROSPERO CRD42016042391).

As a post hoc analysis, we have now examined the composition of diets used by the control group to characterize diets advised for treatment of GDM in usual clinical care. Data on ten dietary characteristics (kcal/day, \% of energy provided by carbohydrates, protein, fat, monounsaturated fat, saturated fat and polyunsaturated fat, grams of fiber/day, glycemic index and load) were collected. Glycemic index is defined as the incremental area under the blood glucose curve following the ingestion of a test food, expressed as percentage of the corresponding area following an equivalent load of a reference carbohydrate. The glycemic load takes into account the amount of food intake.

We have used STATA 14.0 and a random effects model to pool the diet characteristics. Heterogeneity was assessed using $I^{2}$ statistics and Cochran's $Q$ test. A figure displaying worldwide carbohydrate energy contribution was constructed using the carbohydrate intake of studies providing this information (filled circle) and carbohydrate advice (open circle) when intake was not available. 
Table 1 Characteristics of control diet in RCTs addressing modified dietary interventions estimates) for GDM treatment (pooled

\begin{tabular}{lcrrrl}
\hline Characteristic & $N$ studies & Median & CI 95\% & $I^{2}$ & $P$ heterogeneity \\
\hline Energy (Kcal/day) & 10 & 2094.0 & $1931.9-2256$ & 98.1 & $<0.001$ \\
\% of energy from carbohydrates & 12 & 49.1 & $45.1-53.1$ & 98.5 & $<0.001$ \\
\% of energy from proteins & 11 & 19.0 & $17.1-20.9$ & 98.5 & $<0.001$ \\
\% of energy from total fat & 11 & 31.5 & $28.6-34.4$ & 97.7 & $<0.001$ \\
\% of energy from saturated fat & 7 & 9.6 & $8.3-10.8$ & 96.6 & $<0.001$ \\
\% of energy from polyunsaturated fat & 6 & 9.5 & $8.3-10.7$ & 99.2 & $<0.001$ \\
\% of energy from monounsaturated fat & 3 & 10.1 & $6.1-14.1$ & 96.8 & $<0.001$ \\
Glycemic index & 4 & 54.3 & $51.2-57.5$ & 98.1 & $<0.001$ \\
Glycemic load & 3 & 122.3 & $108.1-136.4$ & 94.8 & $<0.001$ \\
Fiber (g/day) & 10 & 21.6 & $18.9-24.2$ & 98.0 & $<0.001$
\end{tabular}

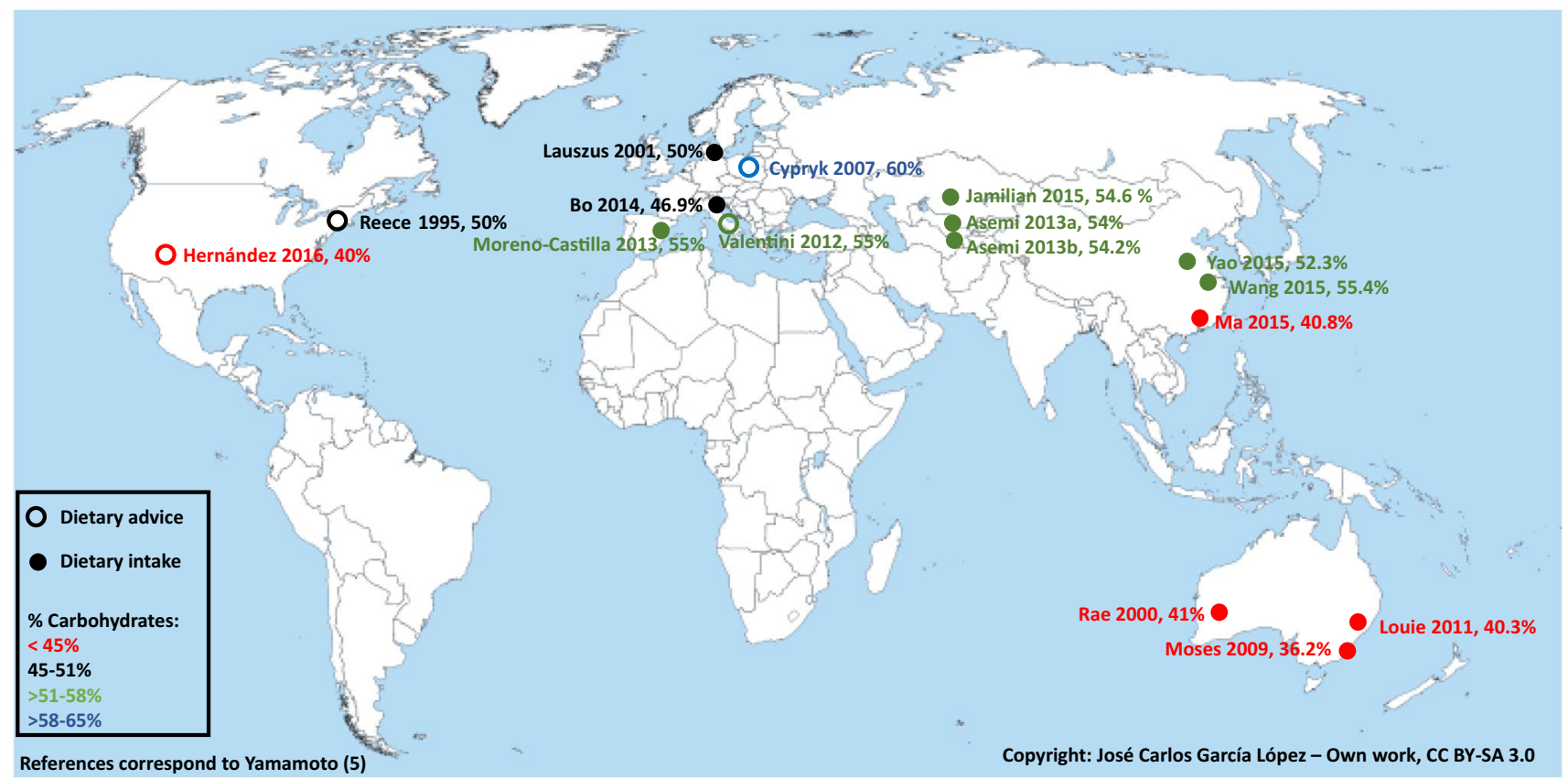

Fig. 1 Energy derived from carbohydrates in control diets of RCTs addressing modified dietary interventions for treatment of GDM by trial site

\section{Results}

Out of 3660 records identified through database search and 128 from other sources, 126 full-text articles were assessed for eligibility and 18 studies were included in the meta-analysis of glycemic control and birthweight-related variables [4]. Thirteen of these studies provided quantitative information on one or more diet characteristics and were included in the current meta-analysis and graphical display. The carbohydrate intake was the diet characteristic most frequently reported $(N=12)$. Other studies only reported diet recommendations and the four of them giving data on carbohydrate advice were included for graphical display.

In the 13 studies included in the current analysis, the modified dietary intervention used for treatment of GDM was as follows: a low glycemic index $\operatorname{diet}(N=4)$, a low carbohydrate diet $(N=1)$, Dietary Approaches to Stop Hypertension $(N=3)$, modification of dietary fat $(N=2)$, soy protein enrichment $(N=1)$, behavioral intervention $(N=1)$, and calorie restriction $(N=1)$. The information in the intervention arm is not used in the current analysis.

Pooled estimates on control diet characteristics are summarized in Table 1. High heterogeneity was observed in the ten diet characteristics ( $I^{2}$ ranging from 94.8 for glycemic load to 99.2 for \% of energy from polyunsaturated fat; $p$ for heterogeneity $<0.001$ for all of them).

The dietary carbohydrate content of control diets in individual trials is displayed in Fig. 1. Carbohydrate contribution to energy intake ranged from moderate restriction $(36.2 \%$ in Australia) to the upper range of the acceptable macronutrient distribution range $(60.0 \%$, Poland). 


\section{Discussion}

In this subanalysis addressing control diets in RCTs on modified dietary interventions for GDM, we observed a high heterogeneity in the ten analyzed characteristics. This information has not been previously reported.

The figures of carbohydrate content of control diets parallel with some exceptions the diet composition in the background population according to FAO statistics [5] with the incorporation of some degree of carbohydrate restriction.

It is of note that specific dietary recommendations with regard to energy-yielding nutrients are lacking for treatment of GDM. A limitation of the current analysis is that we did not perform a specific systematic review and meta-analysis to address this topic but a subanalysis of a previous one [4]. However, current results can serve as an estimation of diets usually advised to women with GDM. Another limitation is that the number of meals and snacks was not addressed.

We conclude that control diets used in RCTs addressing modified dietary intervention in women with GDM display marked heterogeneity in all analyzed characteristics, probably reflecting the diet properties of the background population. This is desirable from the cultural and socioeconomic point of view, but may have an impact on the response to nutritional management of GDM and should be addressed in future research.

Funding HRM was funded by the UK National Institute for Health Research (CDF 2013-06-035). This work was conducted by an expert group of the European branch of the International Life Sciences Institute, ILSI Europe. This publication was coordinated by the Early Nutrition and Long-Term Health and the Obesity and Diabetes Task Forces. Industry members of this task force are listed on the ILSI Europe website at http://www.ilsi.eu/. Experts are not paid for the time spent on this work; however, the non-industry members within the expert group were offered support for travel and accommodation costs from the Early Nutrition and Long-Term Health and the Obesity and Diabetes Task Forces to attend meetings to discuss the manuscript and a small compensatory sum (honoraria) with the option to decline. The expert group carried out the work, i.e., collecting/analyzing data/information and writing the scientific paper apart from other activities of the task forces. The research reported is the result of a scientific evaluation in line with ILSI Europe's framework to provide a precompetitive setting for public-private partnership (PPP). ILSI Europe facilitated scientific meetings and coordinated the overall project management and administrative tasks relating to the completion of this work. The opinions expressed herein and the conclusions of this publication are those of the authors and do not necessarily represent the views of ILSI Europe or those of its member companies. For further information about ILSI Europe, please emailinfo@ilsieurope.be or call+3227710014.

\section{Compliance with ethical standards}

Conflict of interest EMvdB works part-time for Danone Nutricia. RR works full-time for Abbot Nutrition. ECG works full-time for Nestec. All other authors declare that they have no conflict of interest.

Statement of Human and Animal Rights Not applicable, the study is a systematic review and meta-analysis.

Informed consent For this type of study, formal consent is not required.

Open Access This article is distributed under the terms of the Creative Commons Attribution 4.0 International License (http://creativeco mmons.org/licenses/by/4.0/), which permits unrestricted use, distribution, and reproduction in any medium, provided you give appropriate credit to the original author(s) and the source, provide a link to the Creative Commons license, and indicate if changes were made.

\section{References}

1. Meek CL, Lewis HB, Patient C, Murphy HR, Simmons D (2015) Diagnosis of gestational diabetes mellitus: falling through the net. Diabetologia 58:2003-2012. https://doi.org/10.1007/s0012 5-015-3647-z

2. Egan AM, Vellinga A, Harreiter J et al (2017) Epidemiology of gestational diabetes mellitus according to IADPSG/WHO 2013 criteria among obese pregnant women in Europe. Diabetologia 60:1913-1921. https://doi.org/10.1007/s00125-017-4353-9

3. Metzger BE, Buchanan TA, Coustan DR,et al (2007) Summary and Recommendations of the Fifth International Workshop-Conference on Gestational Diabetes Mellitus. Diabetes Care 30:S251260. https://doi.org/10.2337/dc07-s225

4. Yamamoto JM, Kellett JE, Balsells M et al (2018) Gestational diabetes mellitus and diet: a systematic review and meta-analysis of randomized controlled trials examining the impact of modified dietary interventions on maternal glucose control and neonatal birth weight. Diabetes Care 41:1346-1361. https://doi. org/10.2337/dc18-0102

5. FAO. ChartsBin statistics collector team 2011, Contribution of Carbohydrates in Total Dietary Consumption, ChartsBin.com, viewed 6th May, 2018, http://chartsbin.com/view/1154 


\section{Affiliation}

\section{Apolonia García-Patterson ${ }^{1}$ - Montserrat Balsells ${ }^{2}$ - Jennifer M. Yamamoto ${ }^{3}$. Joanne E. Kellett ${ }^{4}$. Ivan Solà',5,6 . Ignasi Gich ${ }^{6,7,8,9}$. Eline M. van der Beek ${ }^{10,11}$ - Eran Hadar ${ }^{12}$. Eurídice Castañeda-Gutiérrez ${ }^{13}$. Seppo Heinonen ${ }^{14,15}$. Moshe $\mathrm{Hod}^{12}$. Kirsi Laitinen ${ }^{16,17}$. Sjurdur F. Olsen ${ }^{18}$. Lucilla Poston ${ }^{19}$. Ricardo Rueda ${ }^{20}$. Petra Rust ${ }^{21}$. Lilou van Lieshout ${ }^{22} \cdot$ Bettina Schelkle ${ }^{22} \cdot$ Helen R. Murphy ${ }^{4,23,24} \cdot$ Rosa Corcoy $^{25,26,27}$}

1 Institute of Biomedical Research (IIB Sant Pau), Hospital de la Santa Creu i Sant Pau, Barcelona, Spain

2 Department of Endocrinology and Nutrition, Hospital Mútua de Terrassa, Terrassa, Spain

3 Division of Endocrinology, Department of Medicine, University of Calgary, Calgary, Canada

4 Norfolk and Norwich University Hospitals, Norfolk, UK

5 Iberoamerican Cochrane Centre, Hospital de la Santa Creu i Sant Pau, Barcelona, Spain

6 CIBER Epidemiología y Salud Pública (CIBERESP), Instituto de Salud Carlos III, Madrid, Spain

7 Department of Epidemiology, Hospital de la Santa Creu i Sant Pau, Barcelona, Spain

8 Department of Pharmacology and Therapeutics, Universitat Autònoma de Barcelona, Bellaterra, Barcelona, Spain

9 CIBER Salud Mental (CIBERSAM), Instituto de Salud Carlos III, Madrid, Spain

10 Nutricia Research, Utrecht, The Netherlands

11 Department of Pediatrics, University Medical Centre Groningen, University of Groningen, Groningen, The Netherlands

12 Rabin Medical Center, Tel-Aviv University, Tel-Aviv, Israel

13 Nestlé Research Center, Lausanne, Switzerland
14 Obstetrics and Gynecology, University of Helsinki, Helsinki, Finland

15 Helsinki University Hospital, Helsinki, Finland

16 Institute of Biomedicine, University of Turku, Turku, Finland

17 Turku University Hospital, Turku, Finland

18 Statens Serum Institut, Copenhagen, Denmark

19 King's College, London, UK

20 R\&D Department, Abbott Nutrition, Granada, Spain

21 Department of Nutritional Sciences, University of Vienna, Vienna, Austria

22 ILSI Europe a.i.s.b.l., Brussels, Belgium

23 Cambridge University Hospitals NHS Foundation Trust, Cambridge, UK

24 Norwich Medical School, University of East Anglia, Norwich, UK

25 Department of Medicine, Universitat Autònoma de Barcelona, Bellaterra, Barcelona, Spain

26 CIBER Bioengineering, Biomaterials and Nanotechnology (CIBER-BBN), Instituto de Salud Carlos III, Madrid, Spain

27 Servei d'Endocrinologia i Nutrició, Hospital de la Santa Creu i Sant Pau, Sant Antoni M Claret 167, 08025 Barcelona, Spain 